\title{
Imaging the human motor system's beta-band synchronization during isometric contraction
}

\author{
Jan-Mathijs Schoffelen, ${ }^{a, *}$ Robert Oostenveld, ${ }^{a}$ and Pascal Fries ${ }^{\mathrm{a}, \mathrm{b}}$ \\ ${ }^{\mathrm{a}}$ F.C. Donders Centre for Cognitive Neuroimaging, Nijmegen, The Netherlands \\ ${ }^{\mathrm{b}}$ Department of Biophysics, Radboud University Nijmegen, The Netherlands
}

Received 20 June 2007; revised 13 December 2007; accepted 7 January 2008

Available online 12 February 2008

Rhythmic synchronization likely subserves interactions among neuronal groups. One of the best studied rhythmic synchronization phenomena in the human nervous system is the beta-band $(15-30 \mathrm{~Hz})$ synchronization in the motor system. In this study, we imaged structures across the human brain that are synchronized to the motor system's beta rhythm. We recorded whole-head magnetoencephalograms (MEG) and electromyograms (EMG) of left/right extensor carpi radialis muscle during left/right wrist extension. We analyzed coherence, on the one hand between the EMG and neuronal sources in the brain, and on the other hand between different brain sources, using a spatial filtering approach. Cortico-muscular coherence analysis revealed a spatial maximum of coherence to the muscle in motor cortex contralateral to the muscle in accordance with earlier findings. Moreover, by applying a two-dipole source model, we unveiled significantly coherent clusters of voxels in the ipsilateral cerebellar hemisphere and ipsilateral cerebral motor regions. The spatial pattern of coherence to the right and left arm EMG was roughly mirror reversed across the midline, in agreement with known physiology. Subsequently, we analyzed the brain-wide pattern of beta-band coherence to the motor cortex contralateral to the contracting muscle. This analysis did not reveal any convincing pattern. Because the prior cortico-muscular analysis had demonstrated the expected pattern in our data, this negative finding demonstrates a current limitation of the applied method for cortico-cortical coherence analysis. We conclude that during an isometric muscle contraction, several distributed brain regions form a brain-wide beta-band network for motor control.

(C) 2008 Elsevier Inc. All rights reserved.

Abbreviations: DICS, dynamic imaging of coherent sources; ECR, extensor carpi radialis.

* Corresponding author. Present address: Centre for Cognitive Neuroimaging in Glasgow, Dept of Psychology, University of Glasgow, Glasgow, UK, G12 8QW. Fax: +44 1413304606.

E-mail address: j.schoffelen@psy.gla.ac.uk (J.-M. Schoffelen).

Available online on ScienceDirect (www.sciencedirect.com).

\section{Introduction}

Oscillatory activity in the beta frequency band $(15-30 \mathrm{~Hz})$ is a conspicuous aspect of neuronal activity in the sensorimotor system. Numerous studies have documented beta-band synchronization within and between several brain structures involved in motor control. Local beta-band synchronization has been shown in primary motor cortex (Murthy and Fetz, 1996), the cerebellum (Courtemanche et al., 2002) and the basal ganglia (Courtemanche et al., 2003). Betaband coherence has been described between primary motor cortex and spinal cord (Conway et al., 1995; Brown, 2000; Kilner et al., 2000; Baker et al., 1997), between cerebellum and motor cortex (Courtemanche and Lamarre, 2005; Soteropoulos and Baker, 2006), between cerebellum and spinal cord (Aumann and Fetz, 2004), between motor cortex and thalamus (Marsden et al., 2000), between spinal cord and thalamus (Marsden et al., 2000), between motor cortex and somatosensory cortex (Brovelli et al., 2004; Ohara et al., 2001), between motor cortex and basal ganglia (Marsden et al., 2001), and between bilateral motor cortices (Murthy and Fetz, 1996; Mima et al., 2000).

Thus, there is extensive evidence for a beta-band network for motor control. However, it is not yet clear whether the reported findings can be generalized to the normal human brain. The studies involving deep structures (thalamus, basal ganglia and cerebellum) used recordings either from animals or from patients. In the healthy human brain, studies so far documented only beta-band coherence between the EMG and the contralateral motor cortex (Conway et al., 1995; Brown, 2000; Kilner et al., 2000) and between the two motor cortices (Mima et al., 2000). In human subjects, non-invasive approaches to uncovering beta-band coherence between different brain structures have to put up with the problem of volume conduction (Nunez et al., 1997). Studies investigating beta-band coherence of brain structures to the EMG circumvent this, but have so far only documented coherence to the contralateral motor cortex. This raises the important question of whether in normal human subjects, the beta-band coherence between other motor control structures can be shown with non-invasive recording techniques.

To address this issue, we investigated cortico-muscular and cortico-cortical beta-band coherence in normal human subjects 
while they maintained an isometric extension of one of their wrists. Coherence was assessed by using combined EMG and MEG recordings and spatial filtering for source estimation. In the spatial filtering approach, neuronal activity is estimated by scanning the brain volume, which for this purpose is divided into a grid of target positions. For each of these positions, a spatial filter is created. This spatial filter is then used to estimate the neuronal activity originating from this given location, and ideally only passes the activity from this target location while suppressing the activity coming from all other potential source locations. Subsequently, coherence can be computed, either between a location in the brain and the EMG, or between two locations within the brain.

We used the frequency domain version of spatial filtering that directly estimates sources of oscillatory MEG activity, the DICS algorithm (Dynamic Imaging of Coherent Sources (Gross et al., 2001)). DICS has been used with great success in several studies that identified coherent networks of brain regions involved in motor activity, both in healthy subjects and in patients with movement disorders (Gross et al., 2002; Timmermann et al., 2003b,a; Pollok et al., 2005b,a; Gross et al., 2004, 2005). However, the frequency at which these synchronized networks oscillated corresponded either to the frequency of pathological or voluntary tremor or to the alpha band $(8-12 \mathrm{~Hz})$, which has an extraordinarily high signal-to-noise ratio.

Despite these successful accounts of cortico-cortical networks, imaging of coherent sources in the brain remains difficult. In particular, the adaptive spatial filtering algorithm is known to perform suboptimally in the presence of correlated sources (Van Veen et al., 1997). Furthermore, the interpretation of coherence among estimated sources is complicated because the signals measured outside the head constitute mixtures of the physiological signal sources and unmixing will never be perfect. Part of these difficulties could be circumvented by using a multiple dipole model for the estimation of the source activity. Such a modified source model has been applied successfully to accurately localize bilateral auditory evoked fields, using time domain beamformers (Dalal et al., 2006; Brookes et al., 2007). We took a similar approach, but now to analyze sources and their interactions in the frequency domain.

The original DICS algorithm uses a single dipole as the underlying model to estimate source activity and is thus susceptible to the presence of correlated sources. We used a modified DICS algorithm that uses a two-dipole source model. This approach is very similar to the beamformer approach proposed in Dalal et al. (2006) and Brookes et al. (2007). We placed one dipole into contralateral motor cortex and assessed coherence of the EMG to the other dipole, which scanned the entire brain volume. With this approach, we found betaband coherence of the EMG not only to motor cortex contralateral to the studied muscle, but also to ipsilateral motor cortex and ipsilateral cerebellum. We performed this experiment for wrist extensions of either arm and found that the resulting patterns of brain-wide coherence to the respective EMG roughly switched over into the other hemisphere, consistent with known physiology.

Subsequently, we fixed a reference dipole in the motor cortex contralateral to the involved muscle and analyzed coherence of this dipole to the rest of the brain. The cortico-muscular analysis had revealed that a beta-band network with several spatially distinct peaks is present in the dataset. We tested whether this network, and possibly additional network nodes, could also be revealed in the cortico-cortical analysis. However, no convincing distant peak of coherence to the reference dipole could be demonstrated. This held true whether coherence was determined between two independently estimated dipoles or between two simultaneously estimated dipoles that did not suppress each other. This negative finding illustrates that cortico-cortical coherence analysis, at least with the methods used in this study, still faces severe limitations.

\section{Materials and methods}

\section{Subjects}

Eighteen healthy subjects (mean age: 25.7 years (range 2132 years), 6 female, 5 left-handed) took part in the experiment. All subjects gave written informed consent according to the Declaration of Helsinki.

\section{Experimental paradigm}

The subjects were seated comfortably with their forearms in a relaxed position, supported by cushions on the armrests of the MEGchair. They were asked to fixate on a dot which was projected on a screen in front of them. The subject's task was to extend one of his/ her wrists against the lever of a force meter and to keep the measured force within a specified window, which was defined between 1.1 and 1.5 N. Fourteen of the subjects received feedback about the exerted force through the color of the fixation dot, which turned from red to green as soon as the required force level was reached. The color change marked the beginning of a trial. A typical trial lasted $7 \mathrm{~s}$, but was aborted as soon as the applied force drifted out of the specified window. The remaining four subjects were asked to extend their wrist against a lever and to keep their hand in a fixed position. They were not given feedback about the force applied. The subjects performed two blocks of trials, one for each side. There was a 2second intertrial interval, in which the subjects were instructed to relax their wrist and to blink.

\section{Data acquisition}

Force applied to the lever was measured by strain gauges. Apart from being recorded in parallel with the electrophysiological data, the force signal was also fed into a separate A/D-converter and a software window discriminator was implemented to detect whether the force was within the specified window.

MEG was acquired with a 151-sensor axial gradiometer system (Omega 2000 whole head MEG-system, CTF systems Inc., Port Coquitlam, Canada). Bipolar surface EMG was recorded from the right and left extensor carpi radialis longus muscles using $2 \mathrm{Ag} /$ $\mathrm{AgCl}$ electrodes, which were placed over the muscles with a $3-\mathrm{cm}$ interelectrode distance, with the proximal electrodes placed $4 \mathrm{~cm}$ distal to the external epicondyle of the humerus. The EOG was recorded from a bipolar electrode pair placed above and lateral to the outer canthus of the left eye. The impedance of the EMG and EOG electrodes was below $20 \mathrm{k} \Omega$. The data were low-pass filtered at $300 \mathrm{~Hz}$ and digitized at $1200 \mathrm{~Hz}$. Prior to and after the MEG recording, the subject's head position relative to the gradiometer array was determined using coils positioned at the subject's nasion, and at the bilateral external auditory meatus.

\section{EMG/MEG preprocessing}

All analyses were done with FieldTrip, an open source Matlabtoolbox for neurophysiological data analysis (F.C. Donders Centre 
for Cognitive Neuroimaging, Nijmegen, The Netherlands; http:// www.ru.nl/fcdonders/fieldtrip).

Only data segments between $1 \mathrm{~s}$ after the start of a trial and trial offset were analyzed. The data were cut into 1-second epochs. Segments that were contaminated by eye movements, muscle activity or jump artifacts in the MEG sensors were discarded. The line noise was removed by estimating and subtracting the $50 \mathrm{~Hz}, 100 \mathrm{~Hz}$ and $150 \mathrm{~Hz}$ components in the MEG data, using a discrete Fourier transform.

The EMG-amplitude was estimated by high-pass filtering the raw EMG at $10 \mathrm{~Hz}$ and taking the absolute value of the Hilberttransformed signal. This procedure gives a time-dependent estimate of the firing rate of the muscular units. It is equivalent to the commonly used full rectification of the EMG signal (Myers et al., 2003). In the following we will use the term "EMG" for this preprocessed EMG signal.

\section{Spectral analysis}

Coherence spectra between all MEG signals and the EMG were computed using the multitaper method (Mitra and Pesaran, 1999). Each data epoch was tapered using a set of discrete prolate spheroidal sequences (Slepian functions). The number of tapers used determines the amount of spectral smoothing. Each tapered data epoch was Fourier-transformed, and auto- and cross-spectra were computed. Subsequently, the spectra were averaged over tapers and data epochs, and the coherence was calculated.

To maximize the sensitivity of subsequent analysis steps, we determined the peak frequency and the amount of spectral smoothing for each subject and condition separately. To this end, we computed the coherence between an EMG signal and all MEG signals. For different amounts of spectral smoothing, the width of the coherence peak was determined by visual inspection. Subsequently, we selected the coherence spectrum in which the width of the peak was approximately equal to the smoothing bandwith. From this spectrum, we defined the peak frequency as the center of the coherence peak.

\section{Single subject source analysis}

For the analysis of the neuronal sources, we used DICS (Gross et al., 2001). In this approach, DICS is used to first estimate source activity irrespective of the EMG and subsequently, the coherence between the estimated source activity and the EMG is determined. This step in the analysis typically yields a spatial maximum of coherence, and the identified brain region can subsequently be used as a seed region for cortico-cortical coherence analysis (Gross et al., 2001).

In order to estimate the source activity, each subject's brain volume was divided into a regular $7.5 \mathrm{~mm}$ grid and for each grid location, a spatial filter was constructed. This filter has the property that it optimally passes activity from the location of interest, while other activity that is present in the data is suppressed. The filter weights are calculated with the following formula:

$w(r, f)=\left(L^{\prime}(r)(C(f)+\lambda \mathbf{I})^{-1} L(r)\right)^{-1} L^{\prime}(r)(C(f)+\lambda \mathbf{I})^{-1}$

Where $L(r)$ is the forward model for the location $r$ of interest (the leadfield matrix), $C(f)$ is the cross-spectral density (CSD) matrix between all MEG signal pairs at the frequency $f, \lambda$ is a regularization parameter, and $\mathbf{I}$ is the identity matrix.
To compute the leadfield matrix, we used a multisphere model in which, for each sensor, a sphere was fitted to the inside of the skull underlying that sensor (Huang et al., 1999). The inside of the skull was derived from each individual subject's structural MRI, which was spatially coregistered with the MEG sensors. In a multisphere model there is no truly magnetically silent direction, in contrast to a single-sphere model, where the description of the leadfield at a given location only requires two tangential dipole orientations. This is because the radial orientation for a sphere fitted to one gradiometer will be different from the radial orientation for a sphere fitted to another gradiometer. As such, there is no truly silent radial direction, and the leadfield comprises three columns. However, the dipole orientation towards the skull surface results in a contribution to the leadfield which is very small (i.e. "almost silent"). In the computation of the filter weights (in which the leadfield is inverted), this potentially results in an inflated contribution to the filter weights. When the data are projected through the spatial filters, noise in the data is subsequently amplified in this almost silent dipole direction. Therefore, we excluded the most silent direction from the leadfields by a singular value decomposition of the leadfield matrix followed by a reconstruction after setting the smallest singular value to 0 .

The CSD matrix between all MEG sensor pairs was computed from the Fourier transforms of the tapered data epochs at the frequency of interest, using an optimized amount of spectral smoothing.

Spatial filters were constructed for each grid location and the Fourier transforms of the tapered data epochs were projected through the spatial filters, using the following formula:

$\mathbf{d}(r, f)=w(r, f) \mathbf{F}(f)$

where $\mathbf{F}$ is an $N \times M$ matrix, containing the Fourier coefficients at frequency $f$, of $N$ channels and $M$ single tapered data epochs. The matrix d represents the Fourier coefficients in three orthogonal directions $(3 \times M$ matrix $)$ for a dipole at location $r$. We estimated the orientation in which the power of the dipole was maximal by applying a singular value decomposition to the real part of the cross-spectral density of the dipole and projected the Fourier coefficients on this direction, yielding a $1 \times M$ FFT vector. This vector represents the Fourier coefficients for the dipole with an orientation in the direction of maximal power. Coherence between each grid location and the EMG could then be computed according to:

$$
\operatorname{Coh}(r, f)=\frac{\left|F_{\text {dipole }}(r, f)^{*} F_{\mathrm{EMG}}(f)\right|^{2}}{\left(F_{\text {dipole }}(r, f)^{*} F_{\text {dipole }}(r, f)\right) \times\left(F_{\mathrm{EMG}}(f)^{*} F_{\mathrm{EMG}}(f)\right)}
$$

where $F_{\text {dipole }}(r, f)$ is the estimated dipole activity at location $r$ and frequency $f, F_{\mathrm{EMG}}(f)$ is the Fourier transform of the EMG at frequency $f$, and * denotes conjugate transposition.

To assess the presence of other brain regions participating in a beta-coherent network, we identified the spatial maximum of cortico-muscular coherence, i.e. the region in motor cortex contralateral to the involved muscle that exhibited the largest coherence to the muscle. We then used this spatial maximum as a seed voxel for cortico-cortical coherence analysis. We computed coherence from this voxel to all other voxels by replacing $F_{\text {EMG }}(f)$ in Eq. (3) by the estimated Fourier transforms of the activity at the seed voxel. This typically yields a volumetric image of corticocortical coherence with very high coherence values in the vicinity of the reference voxel. Clearly, coherence between the reference 
voxel and itself always equals 1. As a consequence of spatial leakage, this value drops only slowly as a function of distance to the reference voxel. These inflated coherence values might obscure the potentially true coherence between cortical sources.

Problems due to spatial leakage could potentially be alleviated by performing a statistical comparison with an appropriate baseline condition or, in general, by contrasting two experimental conditions. The underlying idea is that high coherence values in the vicinity of the reference location are neutralized by similarly high coherence values in the contrasting condition. Thus, when investigating cortico-cortical coherence to a reference in a given motor cortex, we compared contralateral muscle contraction to ipsilateral muscle contraction.

In addition to the problem of spatial leakage, too high correlation between true sources deteriorates the performance of the beamformer algorithm (Van Veen et al., 1997). This problem could be potentially overcome by using a modified source model for the beamformer reconstruction algorithm.

In a second beamformer analysis we therefore used a double dipole model as a source model. It is important to note that the application of the beamformer algorithm is almost identical to the application of the algorithm with a single dipole as a source model. The only difference is the forward model that consists of two dipoles rather than one. When the DICS method is used with a single dipole model, the assumption is made that there are no two linearly correlated or coherent dipoles. The original description of the DICS method estimated that this assumption is valid if coherence is not too strong and the signal-to-noise ratio is sufficient (Gross et al., 2001). However, by using a double dipole model, we explicitly allow for pairs of coherent dipoles. We scanned the brain with two dipoles simultaneously. The location of one of the dipoles was held fixed and was identified for each subject and condition separately, as the voxel in the contralateral hemisphere displaying the highest coherence with the EMG.

To determine the filter weights, we used a similar approach as Dalal et al. (2006) and Brookes et al. (2007), with two differences. First, we used an estimate of the cross-spectral density matrix obtained with multitapers, instead of an estimate of the covariance matrix. Second, we explicitly included the EMG channel in the leadfield matrix by augmenting the leadfield matrix $\mathbf{L}(r)$ according to:

$\mathbf{L}=\left[\begin{array}{ccc}\mathbf{L}_{1} & \mathbf{L}_{2} & 0 \\ 0 & 0 & 1\end{array}\right]$

where $\mathbf{L}_{1}$ and $\mathbf{L}_{2}$ represent the leadfield matrices of the two cortical dipoles.

The filter weights were subsequently estimated using Eq. (1). Multiplying the filter weights with the sensor level Fourier coefficients resulted in the single trial-and-taper estimates of the $7 \times N$ Fourier coefficient matrix on the source level, corresponding with the activity of a fixed dipole in contralateral motor cortex, a dipole at that particular grid location, and of the EMG signal. From this matrix, we computed the cross-spectral density between each of the two cortical sources and the EMG channel, and between the two cortical sources.

For each of the dipoles in a dipole pair, we projected the activity onto the direction in which the power was maximal. Subsequently, we computed coherence between the EMG and the "moving" dipole in each of the dipole pairs. This yielded a volumetric image of cortico-muscular coherence while explicitly taking into account the earlier identified coherently active source (the dipole for which the location was kept fixed). In addition, we computed coherence between the dipoles for each of the dipole pairs, yielding a volumetric image of cortico-cortical coherence with respect to the reference dipole.

\section{Statistical analysis}

Statistics were done on the group level. Individual functional volumes were first $Z$-transformed, either according to Jarvis and Mitra (2001) or according to Maris et al. (2007) when a comparison with a 'baseline' was done. Subsequently, these volumes of $Z$-transformed coherence values were aligned to the subject's structural MRI, spatially normalized to a canonical brain, and smoothed with a $8 \mathrm{~mm}$ Gaussian kernel, using SPM2 (http://www. fil.ion.ucl.ac.uk/spm/software/spm2). We used a non-parametric permutation test in combination with cluster-level statistics to assess significance and correct for multiple comparisons (Nichols and Holmes, 2002; Hayasaka and Nichols, 2004).

The null hypothesis that was tested at the voxel level states that the Z-transformed coherence between the EMG and that voxel, accumulated across subjects, is not different from 0 . Under this null hypothesis, flipping the sign of the $Z$-transformed coherence values of a random subset of the subjects before accumulating leads to an alternative observation that belongs to the null distribution. By repeating this procedure multiple times, a reference distribution of the statistic of interest was created for each voxel.

We corrected for multiple comparisons as follows: In the statistical analysis of the single-dipole beamformer, we computed a reference distribution of the statistic of interest by taking for each randomly permuted volume the value at the voxel showing the highest $Z$-transformed coherence. The observed value at each voxel was tested against this reference distribution.

The signal-to-noise ratio in the two-dipole beamformer analysis was substantially lower than in the single-dipole analysis. This can be seen by comparing the colorbars in Figs. 1 and 2. We therefore applied a weighted cluster statistic to correct for multiple comparisons in the two-dipole analysis. For each randomly permuted volume, clusters of neighboring voxels were identified in which the individual voxels' $p$-value, as obtained from the voxel-level reference distribution, exceeded an a priori threshold. For each cluster we computed the weighted cluster mass (Hayasaka and Nichols,
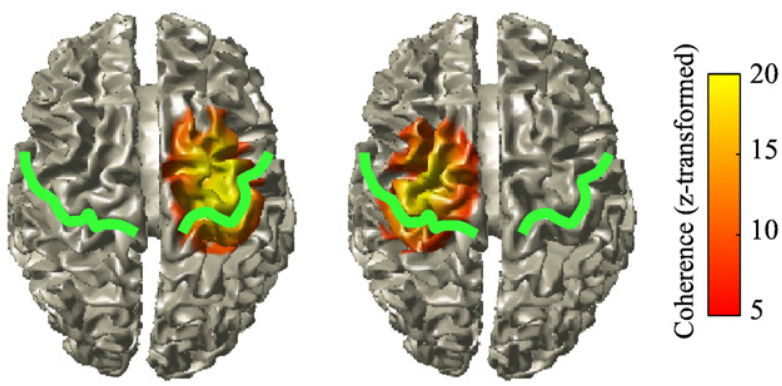

Fig. 1. Source localization of coherence between the EMG and the brain in the beta band, using a single-dipole beamformer. The left panel shows the coherence between the brain and the isometrically contracted left ECR muscle. The right panel shows the coherence between the brain and the isometrically contracted right ECR muscle. Z-transformed coherence was accumulated across subjects that showed a clear coherence peak on the sensor level. Only voxels with a $p$-value $<0.01$ (corrected) are displayed. 


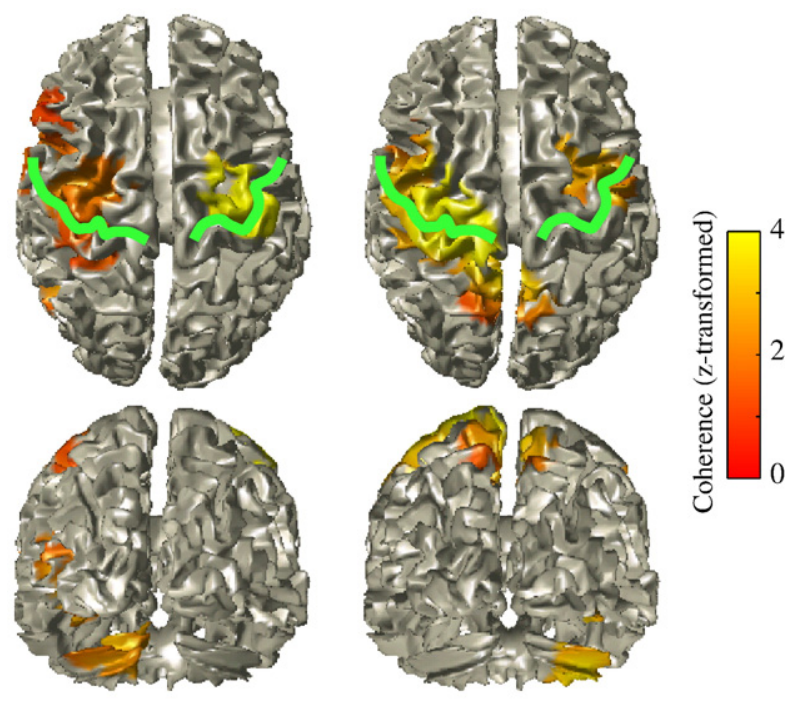

Fig. 2. Source localization of coherence between the EMG and the brain in the beta band, using a two-dipole beamformer. The left panel shows the coherence between the brain and the isometrically contracted left ECR muscle. The right panel shows the coherence between the brain and the isometrically contracted right ECR muscle. Z-transformed cortico-muscular coherence was accumulated across subjects that showed a clear spatial maximum of coherence on the source level. Only voxel clusters with a $p$-value $<0.05$ (corrected) are displayed

2004) and we created a cluster mass reference distribution by taking the "heaviest" cluster for each randomization. The clusters in the observed data were tested against this reference distribution, yielding the probability of observing such a cluster of voxels under the null hypothesis. The choice of the a priori individual voxel threshold determines the shape and spatial extent of the voxel clusters in both the observed and the randomized data. To some extent, this choice is an arbitrary one, and we based the threshold on the observed data for both conditions separately. Our criterion was that spatial clustering of the thresholded voxels in the observed data yielded spatially distinct clusters, instead of few big connected clusters, encompassing large parts of the brain. This yielded an a priori threshold of $p<0.001$ in the condition right and of $p<0.002$ in the condition left. It is important to note that the a priori threshold does not affect the false alarm rate of the statistical test (Nichols and Holmes, 2002).

\section{Estimation of single subject coherence magnitudes and phases}

To get an indication of the magnitude of the coherence in the identified regions, we applied the inverse of the $Z$-transformation to the group results. Since the $Z$-values presented are accumulated values, that is the sum of the single subjects' $Z$-values divided by the square root of $n$, we first divided the group level $Z$-values by $\sqrt{n}$ to obtain the average single subject $Z$-values. For the inverse of the $Z$-transformation, we used an estimate of the degrees of freedom, based on the average degrees of freedom across all subjects. For each significant cluster we subsequently averaged the coherence values across all voxels within each cluster.

To get an indication of the time delay of coherence in the identified regions, we performed the following: For each of the conditions (left and right muscle contraction), we obtained the locations of the three network nodes (contralateral and ipsilateral motor cortex and ipsilateral cerebellum) at the group level in MNIspace by localizing the peak coherence values within each of the nodes. We subsequently inversely warped these coordinates to the individual subjects' brains. Spatial filters were computed for these locations by using a three-dipole source model. For each of the subjects, we used the frequency of maximal cortico-muscular coherence, with a spectral smoothing of $\pm 10 \mathrm{~Hz}$ to compute the filter coefficients. We then projected the orientation of the filters onto the direction of maximally projected power. This yielded, for each dipole position, a set of filter coefficients optimized for a $20 \mathrm{~Hz}$ wide frequency band. Next, we projected the Fouriertransformed sensor level data through these spatial filters to obtain the Fourier coefficients of the reconstructed sources. We restricted this analysis to a $10 \mathrm{~Hz}$ wide frequency band around each subject's optimum frequency, using a multitaper smoothing of $\pm 4 \mathrm{~Hz}$ in steps of $1 \mathrm{~Hz}$. Complex-valued coherency spectra between the Fourier spectrum of the EMG signal, and the Fourier spectra of each of the nodes were computed, and phase difference spectra were obtained. The single subject phase difference spectra were rotated by the mean phase across the analyzed frequency band and averaged across subjects The averaged phase difference spectra were unwrapped to remove the circular ambiguity of the phase difference values, and slopes were fitted, using a least squares fit. Finally, these values were converted to milliseconds by multiplying the estimated slopes with $1000 / 2 \pi$.

\section{Visualization}

For visualization, the clusters of statistically significant voxels were projected onto a high resolution template brain, obtained from the Montreal Neurological Institute (Collins et al., 1998).

\section{Results}

We measured MEG and surface EMG of the right and left extensor carpi radialis muscle (ECR muscle) in 18 subjects, while they exerted a constant force against a lever by extending their wrist. In two blocks of trials, they either had to extend the right wrist (condition right) or the left wrist (condition left). On average, an experimental session yielded 107 (SEM 5.2) 1-second artifactfree data epochs for the condition right and 109 (SEM 7.6) data epochs for the condition left.

During right wrist extension, all 18 subjects showed a peak in the coherence spectrum between the MEG and the right EMG during extension of the right wrist. In the left wrist condition, two of the subjects did not show a coherence peak between the MEG and the left EMG and these were excluded from the subsequent source analysis. The peak frequencies varied across subjects and conditions but were confined to the beta and the low gamma frequency bands (median peak frequency condition right: $25 \mathrm{~Hz}$ (range 19-37 Hz), condition left: $25.5 \mathrm{~Hz}$ (range 20-37 Hz). The mean peak coherence value was 0.028 (range: $0.0031-0.17$ ) in condition right, and 0.034 (range: $0.0022-0.10$ ) in condition left.

Fig. 1 shows the results of the analysis of coherence between the EMG and a single dipole scanned through the brain volume, i.e. the conventional DICS algorithm. The figure shows the projection (onto a template brain) of the statistically significant voxels ( $p<0.01$, corrected for multiple comparisons) that show coherence with the EMG of the active muscle. The panel on the left shows the spatial distribution of coherence to the EMG of the left ECR muscle during left wrist extension. The panel on the right shows 
the respective analysis for condition right. The green line represents the location of the central sulcus. It can be seen that the brain regions that are coherent with the EMG are located in the hemisphere contralateral to the active muscle and anterior to the central sulcus.

The source in contralateral motor cortex with maximal coherence to the muscle was estimated separately for each subject and condition. This voxel was used in subsequent analysis steps. A clear spatial peak of coherence could be identified in 16 subjects in condition right and in 14 subjects in condition left. These subjects were used for subsequent analysis.

Existing evidence suggests that additional brain regions should be synchronized to the motor system's beta-rhythm, but they might remain undetected in our analysis so far because the DICS algorithm suppresses mutually correlated sources. We therefore reestimated neuronal activity with a modified source model. We placed one dipole at the earlier identified seed region and scanned the brain with a second, simultaneously estimated dipole. Subsequently, we computed coherence between the EMG and the "moving" dipole of the dipole pair. Cortico-muscular coherence analysis with the modified source model revealed additional regions beyond contralateral motor cortex that were coherent with the EMG. These regions are shown in Fig. 2. A cluster of voxels could be identified in the cerebellar hemisphere ipsilateral to the active muscle and in the ipsilateral motor cortex. In condition left, there were additional significant clusters in left inferior frontal and left lateral occipital regions and in condition right, there was an additional significant cluster at the parietal midline.

The average magnitude of the coherence between the EMG and the clusters in the motor cortices and the ipsilateral cerebellum is shown in Table 1. Please note that these values are averages over a cluster of voxels that have been determined through a group statistical analysis. This is different from many previous studies that selected the sensor or voxel per subject with the highest coherence and then averaged coherence values across those. To allow for a more direct comparison to this approach, we also included the average (across subjects) cortico-muscular coherence to the voxel in contralateral motor cortex with the highest coherence (determined individually per subject). The average time delay estimated from the cortico-muscular phase spectra is shown in Table 2. For contralateral and ipsilateral cortex, the estimated time delays indicate that the cortex leads the muscle by 6 to $10 \mathrm{~ms}$, consistent with the literature (Riddle and Baker, 2005). For the cerebellum, the estimates were inconsistent across hemispheres and the corresponding phase spectra were noisy.

We also imaged cortico-cortical coherence directly by using the earlier identified seed region as a reference location and by computing coherence between this location and the rest of the brain. Fig. 3 shows the results of this cortico-cortical coherence analysis, using a single

Table 1

Coherence values averaged across the voxels in the significant clusters, and the average maximum coherence value in contralateral motor cortex

\begin{tabular}{lllll}
\hline & $\begin{array}{l}\text { Ipsilateral } \\
\text { cerebellum }\end{array}$ & $\begin{array}{l}\text { Ipsilateral } \\
\text { motor } \\
\text { regions }\end{array}$ & $\begin{array}{l}\text { Contralateral } \\
\text { motor regions } \\
\text { (group data) }\end{array}$ & $\begin{array}{l}\text { Contralateral } \\
\text { motor regions } \\
\text { (individual peaks) }\end{array}$ \\
\hline $\begin{array}{c}\text { Condition } \\
\text { left }\end{array}$ & 0.0013 & 0.0011 & 0.0026 & $0.034(0.0022-0.10)$ \\
$\begin{array}{c}\text { Condition } \\
\text { right }\end{array}$ & 0.0012 & 0.0011 & 0.0014 & $0.028(0.0031-0.17)$ \\
\hline
\end{tabular}

Table 2

Phase delay estimates based on the slope of the phase difference spectra, between the EMG of the contracting muscle, and the three identified brain regions

\begin{tabular}{llll}
\hline & $\begin{array}{l}\text { Ipsilateral } \\
\text { cerebellum }\end{array}$ & $\begin{array}{l}\text { Ipsilateral } \\
\text { motor regions }\end{array}$ & $\begin{array}{l}\text { Contralateral } \\
\text { motor regions }\end{array}$ \\
\hline Condition left & 0.76 & 10 & 8.5 \\
Condition right & 7.4 & 9.2 & 6.0 \\
\hline
\end{tabular}

dipole as a source model. Figs. 3A-D show glass brain projections and a cortical surface projection for condition left, and Figs. 3E-H show the results of the same analysis for condition right. The $Z$-transformed cortico-cortical coherence values are thresholded in two ways: The two columns on the left show 'glass-brain' projections of all voxels with an uncorrected $p$-value $<0.005$. This $p$-value corresponded with the a priori threshold used to identify clusters in the cortico-muscular analysis. The second column from the left shows the same data, with a clipped color scaling, to reveal additional structure in the data. The third column from the left shows 'glass-brain' projections of all voxels with a corrected $p$-value $<$ 0.05 (non-parametric permutation test). To facilitate the comparison with the cortical surface projections in Figs. 1 and 2, the rightmost column shows the projection of the cortico-cortical coherence maps onto the cortical surface, clipped at a value of 10 .

We observed the following:

1 Using a liberal statistical test (first two columns from the left), almost the entire brain shows significant coherence to the reference location. This is likely due to volume conduction effects.

2 Using a conservative statistical test (panels $\mathrm{C}, \mathrm{G}$ ), only the voxels around the reference location become 'significant'. There is no evidence of any distant peak of coherence with the reference in the motor cortex.

3 Some spatial structure beyond the main lobe around the reference position seems to be present in the data and this becomes clear when the color scale is adjusted (panels B, F). However, we suspect that this spatial structure is an artifact. The reason for this suspicion is that this spatial structure is almost precisely symmetric around the reference location as is further demonstrated by panel I, in which an oblique crosscuts is shown, according to the line in panel F. This crosscut shows "sidelobes", which are symmetrically located with respect to the central peak.

Figs. 3J and $\mathrm{K}$ show the difference in cortico-cortical coherence between the active condition (contralateral muscle contraction) and the 'baseline'-condition (ipsilateral muscle contraction). Panel J shows the pooled difference in $Z$-transformed coherence for a reference dipole in the left motor cortex, and panel $K$ for one in the right motor cortex. No mask has been applied to reveal the full spatial structure in the data. Note that the pooled coherence difference in the vicinity of the reference location is not equal to 0 . As expected, the coherence difference at the reference location was 0 for all individual subjects (not shown). However, the shape of the region of high coherence varied across the conditions, which led to nonzero difference values in the voxels neighboring the reference location. Because of the fact that the exact reference location varied across subjects, and because of the spatial smoothing applied, the voxels with a coherence difference of 0 are not visible in the group result. Statistical analysis yielded some clusters of voxels, which 
A

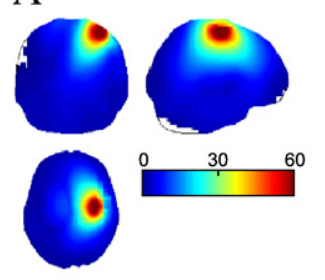

E

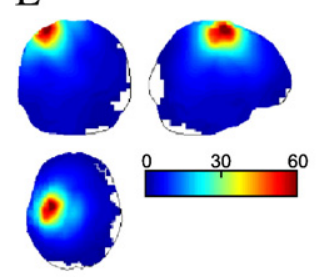

I

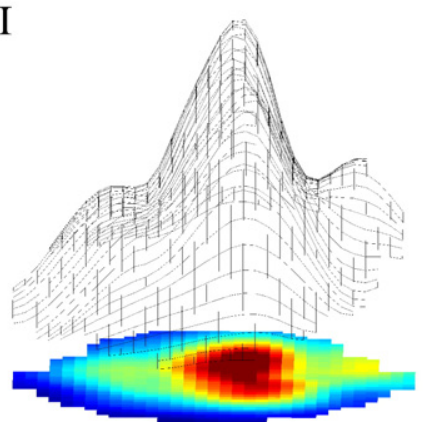

B
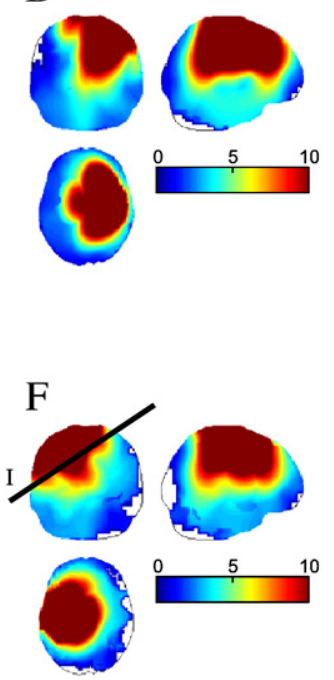

J

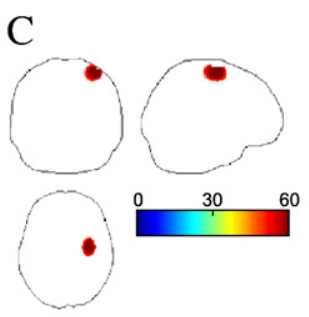

D

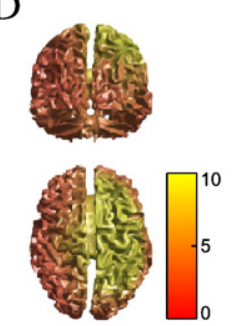

$\mathrm{H}$
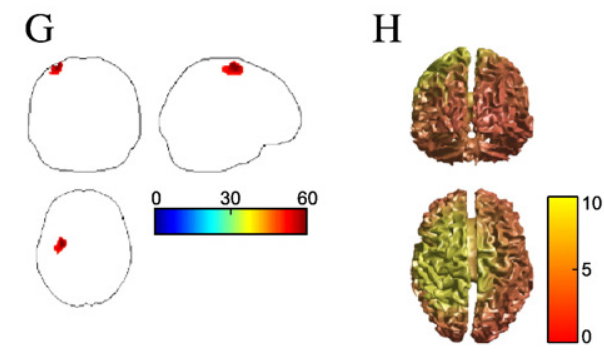

K
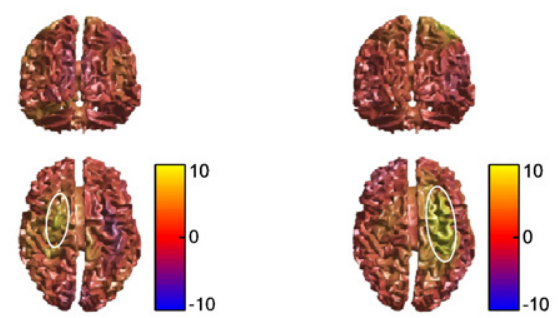

Fig. 3. Cortico-cortical coherence between a dipole in motor cortex contralateral to the activated muscle, and the rest of the brain, using a single dipole model. 'Glass brain' projections of $Z$-transformed cortico-cortical coherence between the right motor cortex and the rest of the brain during contraction of the left ECR muscle $(A-C)$ and between the left motor cortex and the rest of the brain during contraction of the right ECR muscle (E-G), using different statistical masks to threshold the data. (A, B, E, F) Significant voxels at a $p$-value of $<0.005$ (uncorrected) are displayed. Panels B and F show the same results as panels A and E, but with the color range clipped at 10 , to reveal additional spatial structure in the data. (C, G) Only significant voxels at a $p$-value of $<0.05$ (corrected) are displayed. (D, H) The same data as in panel B and F, clipped at a value of 10, projected onto the cortical surface to facilitate comparison with Figs. 1 and 2. (I) A crosscut through the volume displayed in panel F, orthogonal to the coronal plane, and parallel to the black line, to reveal spatial structure in the data. (J, K) Differences in cortico-cortical coherence from a reference dipole to the rest of the brain, between contraction of the contralateral muscle and contraction of the ipsilateral muscle, for a dipole in the left motor cortex $(\mathrm{J})$ and a dipole in the right motor cortex $(\mathrm{K})$. The white ovals represent the suprathreshold clusters of voxels in the vicinity of the reference location.

exceeded the clustering threshold. However, none of these clusters reached significance after multiple comparison correction. The biggest clusters were located in the region of the reference dipole (panel J: $p=0.059$, corrected, panel K: $p=0.054$, white ovals show the approximate location of these clusters). The corresponding coherence differences suggested a tendency for higher local coherence with contralateral muscle contractions, which is most likely explained by a more shallow spatial drop-off of coherence in the contralateral condition.

Using a baseline condition to subtract out the high coherence in the vicinity of the reference dipole relies on the assumption that the shape of the region of high coherence is similar in both conditions. One factor which may severely affect the exact shape of this region is the source power at the reference location. We computed $T$-values of the power difference between the active and baseline conditions in both reference voxels and pooled these $T$-values across subjects. This yielded a $T$-value of 5.5 for the voxel in the left motor cortex ( $p=0.058$, non-parametric randomization test) and a $T$-value of 16.6 for the voxel in right motor cortex $(p<0.0005$, non-parametric randomization test), indicating enhanced beta power during contralateral as compared to ipsilateral contraction. Thus, difference in power at the reference location might explain the differences in cortico-cortical coupling. Therefore, the contrast between corticocortical coherence from two conditions should always be interpreted with care.

The results of the cortico-cortical coherence analysis using the double dipole model are shown in Fig. 4. Statistical analysis of the resulting cortico-cortical coherence volumes yielded essentially the same significant cluster of voxels around the reference dipole, without additional significant clusters of voxels. Also for the double dipole approach, we compared contralateral with ipsilateral muscle contraction (Figs. 4I, J). As for the single dipole case, the biggest clusters were located in the region of the reference dipole, and the cluster around the right motor cortex reference reached significance (panel I (white oval): $p=0.16$, corrected, panel J (opaque cluster of voxels): $p=0.006$ ). In contrast to the single dipole case, the corresponding 
A

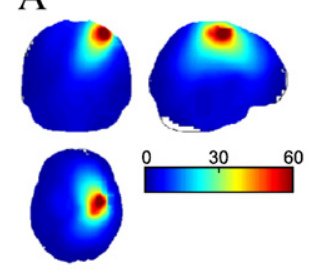

E

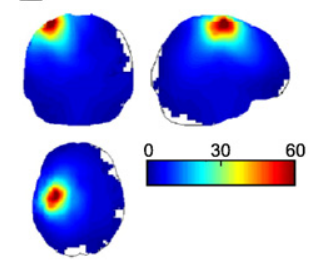

I

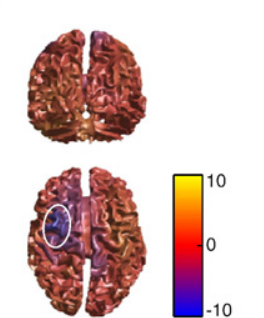

B

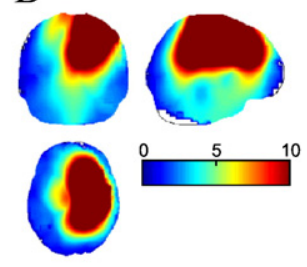

F

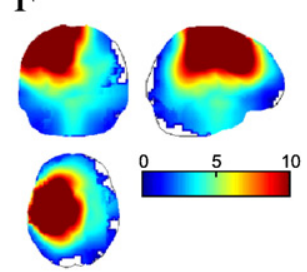

J

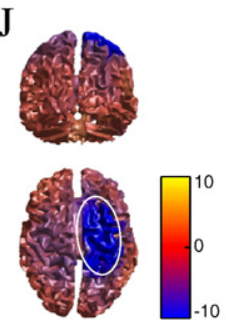

C

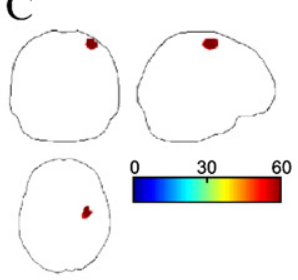

G

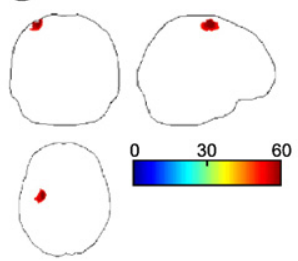

K

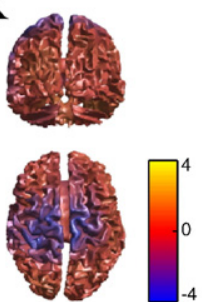

$\mathrm{D}$

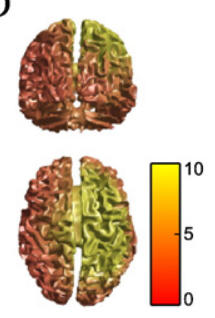

$\mathrm{H}$

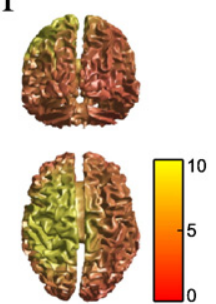

L

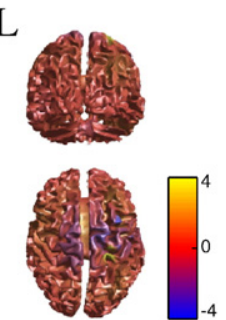

Fig. 4. Cortico-cortical coherence between a dipole in motor cortex contralateral to the activated muscle, and the rest of the brain, using a double dipole model. (A-H) Same conventions as in Figs. 3A-H. (I, J) Same convention as in Figs. 3J-K. (K, L) Difference in cortico-cortical coherence from a reference dipole to the rest of the brain, between a double dipole as a source model, and a single dipole as a source model, for a dipole in the left motor cortex $(\mathrm{K})$ and a dipole in the left motor cortex (L).

coherence differences suggested a tendency for reduced local coherence with contralateral muscle contractions.

The spatial topography of the difference between the coherence maps, as obtained with the two methods, is shown in panel $\mathrm{K}$ (condition left) and L (condition right). Although there was quite some spatial structure in these difference maps, statistical analysis across subjects did not yield significant groups of voxels.

\section{Discussion}

In this study, we used optimized spectral methods and an adaptive spatial filtering algorithm to localize neuronal sources that are beta-coherent during the isometric contraction of a muscle. We confirm several previous studies, reporting EMG to be coherent to the precentral cortex contralateral to the muscle (Conway et al., 1995; Brown, 2000; Kilner et al., 2000; Baker et al., 1997). In addition, we found EMG to be coherent to the cerebellum and the precentral cortex ipsilateral to the muscle. No convincing pattern of cortico-cortical coherence was observed.

The coherence of EMG to ipsilateral structures has so far not been reported in the healthy human brain, despite the fact that it is in good agreement with known physiology and anatomy (Nolte, 1999; Salmelin et al., 1995; Kim et al., 1993). We analyzed the spatial pattern of coherence during right and left wrist extension. Across those two conditions, the spatial pattern of coherence was roughly mirrored across the midline, providing further credibility to the observed patterns. In addition to those regions, a few other regions were coherent with the EMG: The right ECR muscle EMG was coherent with the parietal midline region, and the left ECR muscle EMG was coherent with a left inferior frontal and a left lateral occipital region. Those regions might well be involved in motor control, but likely less directly than the regions in motor cortex and cerebellum. It is therefore not surprising that those additional regions were found less consistently over subjects, each in only one of the two conditions.

As noted above, the coherence that we found to ipsilateral cerebellum is in good agreement with the literature. It is well known that motor control is supported by the cerebellum primarily ipsilateral to the motor effector (Nolte, 1999). Furthermore, microelectrode recordings in awake monkeys documented clear rhythmicity of cerebellar activity: Under certain conditions, neuronal activity in the cerebellum has been found to oscillate strongly in the beta-band (Courtemanche et al., 2002). Beta-band coherence has been demonstrated between the cerebellum and contralateral sensorimotor cortex (Courtemanche and Lamarre, 2005), between cerebellar nuclei and the primary motor cortex (Soteropoulos and Baker, 2006) and between cerebellar nuclei and the ipsilateral EMG (Aumann and Fetz, 2004). The cerebello-muscular coherence found here is in line with these findings.

Also the beta-band coherence of the EMG to ipsilateral motor cortex corresponds well with previous studies. While it is textbook 
knowledge that (distal) motor effectors are primarily controlled by contralateral motor cortex, several studies have documented an involvement of ipsilateral motor cortex. Activation of ipsilateral motor cortex during hand movements has been shown with functional magnetic resonance imaging (fMRI) (Kim et al., 1993; Kobayashi et al., 2003) and MEG (Salmelin et al., 1995). Transcranial magnetic stimulation (TMS) of motor cortex leads to motor evoked potentials (MEPs) not only at contralateral but also at ispilateral (distal) muscles (Ziemann et al., 1999). Rhythmic neuronal activity in the beta-band has been shown in awake monkey sensorimotor cortex both contralateral and ipsilateral to the used motor effector (Murthy and Fetz, 1996). Also, beta-band coherence has been documented between bilateral sensorimotor cortices (Mima et al., 2000; Murthy and Fetz, 1996). Nevertheless, betaband coherence between ipsilateral motor cortex and the muscle has so far not been documented. Indeed, one study explicitly failed to find it (Kilner et al., 2003). However, this study used a bimanual task that precludes a direct comparison with our results.

Several previous studies investigated the brain sources coherent to the muscle in the beta-band (Conway et al., 1995; Kilner et al., 2000, 2003; Baker et al., 1997; Brown, 2000). Those studies reported the muscle to be coherent exclusively to the contralateral sensorimotor cortex. This corresponds to our results when we analyzed coherence between the EMG and a single dipole scanned through the brain volume. We modified this approach as follows: We first determined the source in contralateral motor cortex with strong coherence to the muscle. We then placed one dipole at that location and scanned the brain with a second, simultaneously estimated dipole.

This approach led to two observations.

1.) Overall coherence values decreased as compared to the single-dipole analysis, as can be seen from the different color scales in Figs. 1 and 2.

2.) Despite the overall decrease in coherence values, the modified DICS algorithm unveiled additional regions coherent with the EMG.

Several reasons likely account for this:

1.) The source estimate with the second dipole seemed to lead to an increased effective spatial resolution. When we scanned the brain volume with a single dipole, coherence of contralateral motor cortex extended into a fairly large region around it (not all of this region is visible in the statistically thresholded Fig. 1). The most likely reason for this is that spatial filters created for locations outside contralateral motor cortex did not perfectly avoid leakage of activity from contralateral motor cortex. Spatial leakage of estimated activity generally leads to a poor spatial resolution of the beamformer algorithm. As a consequence of this leakage, activity from contralateral motor cortex spreads to the surrounding voxels. In other words: the estimated activity at a given voxel outside contralateral motor cortex consists of the sum of the true activity at that voxel, and the attenuated activity from elsewhere, including the contralateral motor cortex. Therefore, the estimated phase and amplitude reflect to a variable extent the phase and amplitude of the oscillating dipole in motor cortex. Since the coherence measure is very sensitive to pick up consistent phase differences between signals, even a small amount of leakage will lead to clearly inflated coherence estimates. The modified algorithm seemed to improve this: At the single subject level (data not shown), coherence of the second, scanning dipole was identical to coherence with a single dipole, when the second dipole was in contralateral motor cortex. However, when the second dipole was placed outside contralateral motor cortex, coherence to the EMG was sharply reduced, i.e. leaving a sharp spatial peak of coherence in contralateral motor cortex. This is probably because the first dipole in motor cortex picked up all or most of the local activity, which then did not leak any more into the second dipole. Fig. 2, depicting the coherence of the second dipole to the EMG, does not show this sharp spatial peak in contralateral motor cortex anymore due to spatial smoothing and spatial normalization and averaging across subjects.

2.) Previous invasive studies demonstrated beta-band coherence between different brain regions. The presence of correlated activity is known to degrade the performance of the beamformer (Van Veen et al., 1997; Sekihara et al., 2002). This generally means that when activity is estimated at a given location $\mathrm{A}$, while there is correlated activity originating from another location $\mathrm{B}$, the activity at location A will be underestimated, and vice versa. This mutual suppression is most notable in the presence of strongly coherent sources. But correlated background activity (Sekihara et al., 2002) and low signal-to-noise ratio (Gross et al., 2001) also affect the quality of the beamformer estimate at lower magnitudes of coherence. This most likely does not only affect the estimated strength of the activity, but also affects the estimate of the phase. The phase estimate is a crucial ingredient for the subsequent step, which is the computation of the coherence between the estimated activity at a given brain location and the EMG. Fixing one dipole in contralateral motor cortex and then scanning with a second one leads to an estimate of activity at this second location without the suppressive influence from motor cortex.

Both the coherence of EMG to ipsilateral cerebellum and the coherence to ipsilateral motor cortex could be mediated through direct projections or through an embedding into a larger beta-band network for motor control. This will be an important topic for future studies. It should be noted that the current results demonstrate coherence of the EMG to different brain structures, while not yet providing direct evidence for coherence among those brain structures. Given the overall low coherence values, A and B could be coherent with $\mathrm{C}$, while not being coherent with each other. This would be contrary to what one would expect based on existing intracranial evidence, but it would be possible mathematically. In order to actually test whether the different brain structures, coherent with the EMG, are also coherent with each other, a direct analysis of cortico-cortical coherence is required. However, the cortico-cortical coherence analysis did not yield any distant spatial peaks of coherence with primary motor cortex, most likely due to methodological limitations. The most important limitation is the region of very high coherence values in the vicinity of the reference dipole. These high values might occlude any physiological cortico-cortical coherence. Use of a contrast condition to take into account these high values should be handled with care, due to the confounding influence of power differences across conditions. We found that beta-band power in motor cortex did indeed differ 
significantly between conditions. We also found that the single dipole coherence contrast between contralateral and ipsilateral contraction resulted in a positive cluster around the reference while the same analysis for the double dipole gave a negative cluster. This contradictory result might well be related to an interaction between the different forward models and the power differences at the reference. It will be an important topic for future research to investigate cortico-cortical beta-band coherence with further improved methods.

Several previous studies did successfully analyze cortico-cortical coherence in frequency bands below the beta-band. They also used spatial filters and demonstrated clear cortico-cortical coherence among structures of the motor system in patients with Parkinson disease (Timmermann et al., 2003b) or mini-asterixis (Timmermann et al., 2003a) and in healthy human subjects imitating a tremor or performing finger tapping (Pollok et al., 2005a,b). Also, for slow, smooth finger movements, several motor structures were found to be coherent to each other (Gross et al., 2004, 2002). The frequency at which the respective networks were found to be coherent typically corresponded to the frequency of the overt motor behavior, i.e. the frequency of the tremor $(4-8 \mathrm{~Hz})$ or the velocity fluctuations in smooth finger movements $(6-9 \mathrm{~Hz})$. Some studies of cortico-cortical coherence in the motor system did not rely on the rhythmicity in motor output (Pollok et al., 2005b,a), but rather found corticocortical coherence to occur in the alpha frequency band $(8-12 \mathrm{~Hz})$. The signal-to-noise in these frequency bands is substantially higher than the signal-to-noise ratio of the physiological beta-rhythm of the motor system. With signal-to-noise ratio we denote the relationship between the source signals of interest on the one hand, i.e. the neuronal sources participating in the network, and the background activity and measurement noise on the other hand. The signal-tonoise ratio affects the quality of the source estimate, and leakage of background activity into the estimated source activities prohibits the distinction between leaked coherence and true interaction.

It should be noted that two previous studies reported beta-band cortico-cortical coupling, during rhythmic tapping (Gross et al., 2005), and during a rapid serial visual processing task (Gross et al., 2004). However, neither one of the studies investigated the complete spatial pattern of cortico-cortical coupling, but instead, they both focused on regions-of-interest. These regions were either defined based on the coherence between the brain and the movement frequency during a rhythmic tapping task (Gross et al., 2005) or based on the maxima of stimulus induced beta-band perturbations (Gross et al., 2004). Moreover, in one of these studies (Gross et al., 2004), analysis on phase coupling between these regions-of-interest was only performed on the sensor level.

In this study, we used a beamformer to reconstruct the activity of neuronal sources and their interactions. Recently, a minimum norm estimate has been successfully used to identify long-range cortico-cortical interactions (Jerbi et al., 2007). It will be an interesting target for future research to compare the merits of both localization techniques in the analysis of task-related functional networks.

Our failure to convincingly demonstrate cortico-cortical coherence in the beta-band might well be explained by the lower signalto-noise ratio as compared to earlier successful accounts of corticocortical coherence. As mentioned above, many invasive recordings within and among motor control structures demonstrate the important role of beta-band synchronization (Brovelli et al., 2004; Murthy and Fetz, 1996; Courtemanche et al., 2002, 2003; Soteropoulos and Baker, 2006; Marsden et al., 2000; Ohara et al., 2001) and it is therefore of importance to further explore the potential for studying cortico-cortical coherence in this frequency band non-invasively.

\section{Acknowledgments}

We thank B. Daams for technical assistance and J. Poort for help during part of the experiments. Supported by The Netherlands Organization for Scientific Research, grants 452-03-344 and 05102-050 (P.F.), The Human Frontier Science Program Organization, grant RGP0070/2003 (P.F.), The Volkswagen Foundation, grant I/ 79876 (P.F.) and the Danish Technical Research Council, grant 2601-0092 (R.O.).

\section{References}

Aumann, T.D., Fetz, E.E., 2004. Oscillatory activity in forelimb muscles of behaving monkeys evoked by microstimulation in the cerebellar nuclei. Neurosci. Lett. 361, 106-110.

Baker, S.N., Olivier, E., Lemon, R.N., 1997. Coherent oscillations in monkey motor cortex and hand muscle EMG show task-dependent modulation. J. Physiol. 501, 225-241.

Brookes, M.J., Stevenson, C.M., Barnes, G.R., Hillebrand, A., Simpson, M.I., Francis, S.T., Morris, P.G., 2007. Beamformer reconstruction of correlated sources using a modified source model. Neuroimage 34, 1454-1465.

Brovelli, A., Ding, M., Ledberg, A., Chen, Y., Nakamura, R., Bressler, S.L., 2004. Beta oscillations in a large-scale sensorimotor cortical network: directional influences revealed by Granger causality. Proc. Natl. Acad. Sci. U. S. A. 101, 9849-9854.

Brown, P., 2000. Cortical drives to human muscle: the Piper and related rhythms. Prog. Neurobiol. 60, 97-108.

Collins, D.L., Zijdenbos, A.P., Kollokian, V., Sled, J.G., Kabani, N.J., Holmes, C.J., Evans, A.C., 1998. Design and construction of a realistic digital brain phantom. IEEE Trans. Med. Imag. 17, 463-468.

Conway, B.A., Halliday, D.M., Farmer, S.F., Shahani, U., Maas, P., Weir, A.I., Rosenberg, J.R., 1995. Synchronization between motor cortex and spinal motoneuronal pool during the performance of a maintained motor task in man. J. Physiol. 489, 917-924.

Courtemanche, R., Lamarre, Y., 2005. Local field potential oscillations in primate cerebellar cortex: synchronization with cerebral cortex during active and passive expectancy. J. Neurophysiol. 93, 2039-2052.

Courtemanche, R., Pellerin, J.P., Lamarre, Y., 2002. Local field potential oscillations in primate cerebellar cortex: modulation during active and passive expectancy. J. Neurophysiol. 88, 771-782.

Courtemanche, R., Fujii, N., Graybiel, A.M., 2003. Synchronous, focally modulated beta-band oscillations characterize local field potential activity in the striatum of awake behaving monkeys. J. Neurosci. 23, 11741-11752.

Dalal, S.S., Sekihara, K., Nagarajan, S.S., 2006. Modified beamformers for coherent source region suppression. IEEE Trans. Biomed. Eng. 53, 1357-1363.

Gross, J., Kujala, J., Hämäläinen, M., Timmermann, L., Schnitzler, A., Salmelin, R., 2001. Dynamic imaging of coherent sources: Studying neural interactions in the human brain. Proc. Natl. Acad. Sci. U. S. A. 98, 694-699.

Gross, J., Timmermann, L., Kujala, J., Dirks, M., Schmitz, F., Salmelin, R., Schnitzler, A., 2002. The neural basis of intermittent motor control in humans. Proc. Natl. Acad. Sci. U. S. A. 99, 2299-2302.

Gross, J., Schmitz, F., Schnitzler, I., Kessler, K., Shapiro, K., Hommel, B., Schnitzler, A., 2004. Modulation of long-range neural synchrony reflects temporal limitations of visual attention in humans. Proc. Natl. Acad. Sci. U. S. A. 101, 13050-13055.

Gross, J., Pollok, B., Dirks, M., Timmermann, L., Butz, M., Schnitzler, A., 2005. Task-dependent oscillations during unimanual and bimanual movements in the human primary motor cortex and SMA studied with magnetoencephalography. NeuroImage 26, 91-98. 
Hayasaka, S., Nichols, T.E., 2004. Combining voxel intensity and cluster extent with permutation test framework. NeuroImage 23, 54-63.

Huang, M.X., Mosher, J.C., Leahy, R.M., 1999. A sensor-weighted overlapping-sphere head model and exhaustive head model comparison for MEG. Phys. Med. Biol. 44, 423-440.

Jarvis, M.R., Mitra, P.P., 2001. Sampling properties of the spectrum and coherency of sequences of action potentials. Neural Comput. 13, 717-749.

Jerbi, K., Lachaux, J.P., N'Diaye, K., Pantazis, D., Leahy, R.M., Garnero, L., Baillet, S., 2007. Coherent neural representation of hand speed in humans revealed by MEG imaging. Proc. Natl. Acad. Sci. U. S. A. 104, 7676-7681.

Kilner, J.M., Baker, S.N., Salenius, S., Hari, R., Lemon, R.N., 2000. Human cortical muscle coherence is directly related to specific motor parameters. J. Neurosci. 20, 8838-8845.

Kilner, J.M., Salenius, S., Baker, S.N., Jackson, A., Hari, R., Lemon, R.N., 2003. Task-dependent modulations of cortical oscillatory activity in human subjects during a bimanual precision grip task. NeuroImage 18, 67-73.

Kim, S.G., Ashe, J., Hendrich, K., Ellermann, J.M., Merkle, H., Ugurbil, K., Georgopoulos, A.P., 1993. Functional magnetic resonance imaging of motor cortex: hemispheric asymmetry and handedness. Science 261, 615-617.

Kobayashi, M., Hutchinson, S., Schlaug, G., Pascual-Leone, A., 2003. Ipsilateral motor cortex activation on functional magnetic resonance imaging during unilateral hand movements is related to interhemispheric interactions. NeuroImage 20, 2259-2270.

Maris, E., Schoffelen, J.M., Fries, P., 2007. Nonparametric statistical testing of coherence differences. J. Neurosci. Methods 163, 161-175.

Marsden, J.F., Ashby, P., Limousin-Dowsey, P., Rothwell, J.C., Brown, P., 2000. Coherence between cerebellar thalamus, cortex and muscle in man: cerebellar thalamus interactions. Brain 123 (Pt 7), 1459-1470.

Marsden, J.F., Limousin-Dowsey, P., Ashby, P., Pollak, P., Brown, P., 2001. Subthalamic nucleus, sensorimotor cortex and muscle interrelationships in Parkinson's disease. Brain 124, 378-388.

Mima, T., Matsuoka, T., Hallett, M., 2000. Functional coupling of human right and left cortical motor areas demonstrated with partial coherence analysis. Neurosci. Lett. 287, 93-96.

Mitra, P.P., Pesaran, B., 1999. Analysis of dynamic brain imaging data. Biophys. J. 76, 691-708.

Murthy, V.N., Fetz, E.E., 1996. Oscillatory activity in sensorimotor cortex of awake monkeys: synchronization of local field potentials and relation to behavior. J. Neurophysiol. 76, 3949-3967.

Myers, L.J., Lowery, M., O’Malley, M., Vaughan, C.L., Heneghan, C., St Clair, G.A., Harley, Y.X., Sreenivasan, R., 2003. Rectification and nonlinear pre-processing of EMG signals for cortico-muscular analysis. J. Neurosci. Methods 124, 157-165.

Nichols, T.E., Holmes, A.P., 2002. Nonparametric permutation tests for functional neuroimaging: a primer with examples. Hum. Brain Mapp. 15, 1-25.
Nolte, J., 1999. The Human Brain, An Introduction to its Functional Anatomy, Fourth edition. Mosby, St. Louis.

Nunez, P.L., Srinivasan, R., Westdorp, A.F., Wijesinghe, R.S., Tucker, D.M., Silberstein, R.B., Cadusch, P.J., 1997. EEG coherency. I: Statistics, reference electrode, volume conduction, Laplacians, cortical imaging, and interpretation at multiple scales. Electroencephalogr. Clin. Neurophysiol. 103, 499-515.

Ohara, S., Mima, T., Baba, K., Ikeda, A., Kunieda, T., Matsumoto, R., Yamamoto, J., Matsuhashi, M., Nagamine, T., Hirasawa, K., Hori, T., Mihara, T., Hashimoto, N., Salenius, S., Shibasaki, H., 2001. Increased synchronization of cortical oscillatory activities between human supplementary motor and primary sensorimotor areas during voluntary movements. J. Neurosci. 21, 9377-9386.

Pollok, B., Gross, J., Dirks, M., Timmermann, L., Schnitzler, A., 2004. The cerebral oscillatory network of voluntary tremor. J. Physiol. 554, 871-878.

Pollok, B., Gross, J., Muller, K., Aschersleben, G., Schnitzler, A., 2005a. The cerebral oscillatory network associated with auditorily paced finger movements. NeuroImage 24, 646-655.

Pollok, B., Sudmeyer, M., Gross, J., Schnitzler, A., 2005b. The oscillatory network of simple repetitive bimanual movements. Brain Res. Cogn. Brain Res. 25, 300-311.

Riddle, C.N., Baker, S.N., 2005. Manipulation of peripheral neural feedback loops alters human corticomuscular coherence. J. Physiol. 566, 625-639.

Salmelin, R., Forss, N., Knuutila, J., Hari, R., 1995. Bilateral activation of the human somatomotor cortex by distal hand movements. Electroencephalogr. Clin. Neurophysiol. 95, 444-452.

Sekihara, K., Nagarajan, S.S., Poeppel, D., Marantz, A., 2002. Performance of an MEG adaptive-beamformer technique in the presence of correlated neural activities: effects on signal intensity and time-course estimates. IEEE Trans. Biomed. Eng. 49, 1534-1546.

Soteropoulos, D.S., Baker, S.N., 2006. Cortico-cerebellar coherence during a precision grip task in the monkey. J. Neurophysiol. 95, 1194-1206.

Timmermann, L., Gross, J., Butz, M., Kircheis, G., Haussinger, D., Schnitzler, A., 2003a. Mini-asterixis in hepatic encephalopathy induced by pathologic thalamo-motor-cortical coupling. Neurology 61, 689-692.

Timmermann, L., Gross, J., Dirks, M., Volkmann, J., Freund, H.J., Schnitzler, A., 2003b. The cerebral oscillatory network of parkinsonian resting tremor. Brain 126, 199-212.

Van Veen, B.D., van Drongelen, W., Yuchtman, M., Suzuki, A., 1997. Localization of brain electrical activity via linearly constrained minimum variance spatial filtering. IEEE Trans. Biomed. Eng. 44, 867-880.

Ziemann, U., Ishii, K., Borgheresi, A., Yaseen, Z., Battaglia, F., Hallett, M., Cincotta, M., Wassermann, E.M., 1999. Dissociation of the pathways mediating ipsilateral and contralateral motor-evoked potentials in human hand and arm muscles. J. Physiol. 518 (Pt 3), 895-906. 